\title{
A universal tunable nanofluidic diode via photoresponsive host-guest interactions
}

\author{
Pei Liu' ${ }^{1,2}$, Ganhua Xie ${ }^{1}$, Pei Li ${ }^{3}$, Zhen Zhang ${ }^{1}$, Linsen Yang ${ }^{1}$, Yuanyuan Zhao ${ }^{1}$, Congcong Zhu ${ }^{1,2}$, Xiang-Yu Kong ${ }^{1}$, \\ Lei Jiang ${ }^{1,2,3}$ and Liping Wen ${ }^{1,2,3}$
}

\begin{abstract}
Inspired by biological ion channels, scientists have fabricated various artificial nanosystems. However, nanofluidic diode systems with replaceable functional groups are rarely reported. In this work, we demonstrated a universal tunable nanofluidic diode based on a conical polyimide nanochannel. Using host-guest interactions between $\beta$ cyclodextrin and azobenzene, bidirectional nanofluidic diodes were prepared, and the degree of rectification could be adjusted with high precision by tuning the $\mathrm{pH}$ conditions. This work provides a novel approach to create a nanofluidic platform with replaceable surface functional groups, which has great potential in fields such as photosensitive nanofluidic devices, drug transport and release, and nanofluidic logic devices.
\end{abstract}

\section{Introduction}

Biological ion channels ${ }^{1-3}$ embedded within cell membranes play a significant role in exchanging material, transporting ions, and transforming energy with the extracellular world. ${ }^{4-6}$ Inspired by their genius designs and outstanding properties, scientists have developed various artificial nanosystems ${ }^{7-10}$ that are capable of sensing or separating a diverse variety of ions in aqueous solutions. ${ }^{11,12}$ Recent advances in smart nanofluidic devices in chemistry, materials science, and nanotechnology have gained increasing attention, and nanofluidic diodes, in particular, have evolved quickly. ${ }^{13-17}$ One of the main characteristics of ion transport in these diode-like nanosystems is the ion-rectifying effect. ${ }^{13,14,17,18}$ Like diodes in electronic devices, nanofluidic diodes exhibit ion unidirectional transport behavior owing to several factors, the main of which is the asymmetric distribution of surface

\footnotetext{
Correspondence: Xiang-Yu Kong (kongxiangyu@mail.ipc.ac.cn) or Liping Wen (wen@mail.jpc.ac.cn)

${ }^{1}$ CAS Key Laboratory of Bioinspired Materials and Interfacial Science, Technical Institute of Physics and Chemistry, Chinese Academy of Sciences, Beijing 100190, China

${ }^{2}$ School of Future Technology, University of Chinese Academy of Science, Beijing 100049, China

Full list of author information is available at the end of the article.

These authors contributed equally: Pei Liu, Ganhua Xie
}

charges on the inner surface of nanochannels. ${ }^{17,18}$ The transport direction of ions can be changed by altering the effective surface charge. ${ }^{14,19-21}$ However, existing studies on controlling the ion transport direction in a nanofluidic diode mainly focus on regulating the functional groups, which are normally fixed and non-replaceable, through $\mathrm{pH}^{22-24}$ voltage, ${ }^{15}$ light, ${ }^{25,26}$ ions, ${ }^{14,21,27}$ or other stimuli, ${ }^{28,29}$ resulting in restrictions in changing the type of nanofluidic system as needed. Therefore, fabricating a universal tunable nanofluidic diode $\mathrm{e}^{6,30,31}$ is an interesting and challenging task.

In this work, we demonstrated a $\mathrm{pH}$-regulated bidirectional nanofluidic diode via photoresponsive host-guest interactions based on a conical polyimide (PI) nanochannel. Among addressable nanodevices, lightresponsive nanochannels stand out owing to their reversibility, short response times, and remote control and can potentially be applied in various fields, such as optical information storage and drug release. ${ }^{32-34}$ In our case, light is used as the stimulus to realize the functionalization of the system. As one type of widely used photoresponsive molecule, azobenzene (Azo) groups, change into the trans conformation under irradiation with visible light (vis light, $430 \mathrm{~nm}$ ) and interact with $\beta$-cyclodextrins driven by hydrophobic and van der Waals interactions, 
whereas Azo groups return to the cis conformation under irradiation with ultraviolet light (UV light, $365 \mathrm{~nm}$ ), ${ }^{35,36}$ resulting in a mismatch between the host and guest. $^{30,31,37-40}$ Here, by immobilizing $\beta$-cyclodextrin ( $\beta$ $\mathrm{CD})$ as the "host" and preparing various functionalized Azo-end groups as "guests", a universal tunable system that returns to the original state under different light stimuli can be realized (Scheme 1). ${ }^{38}$ In addition, the $\mathrm{pH}$ responsive functional groups make it possible to regulate the rectification property and perform bidirectional rectification, which can lead to enhanced functionalities of the artificial nanofluidic diode. In addition, the powerful host-guest chemistry has been employed to regulate the wetting behavior and conducting states of the nanopore reversibly with light and electric fields by grafting the Azo group onto the surface of the nanopore. ${ }^{41}$ Based on the reversible and adjustable host-guest interaction, nanofluidic diodes with different surface properties and states can be achieved; thus, various functions can be integrated into a single nanochannel.

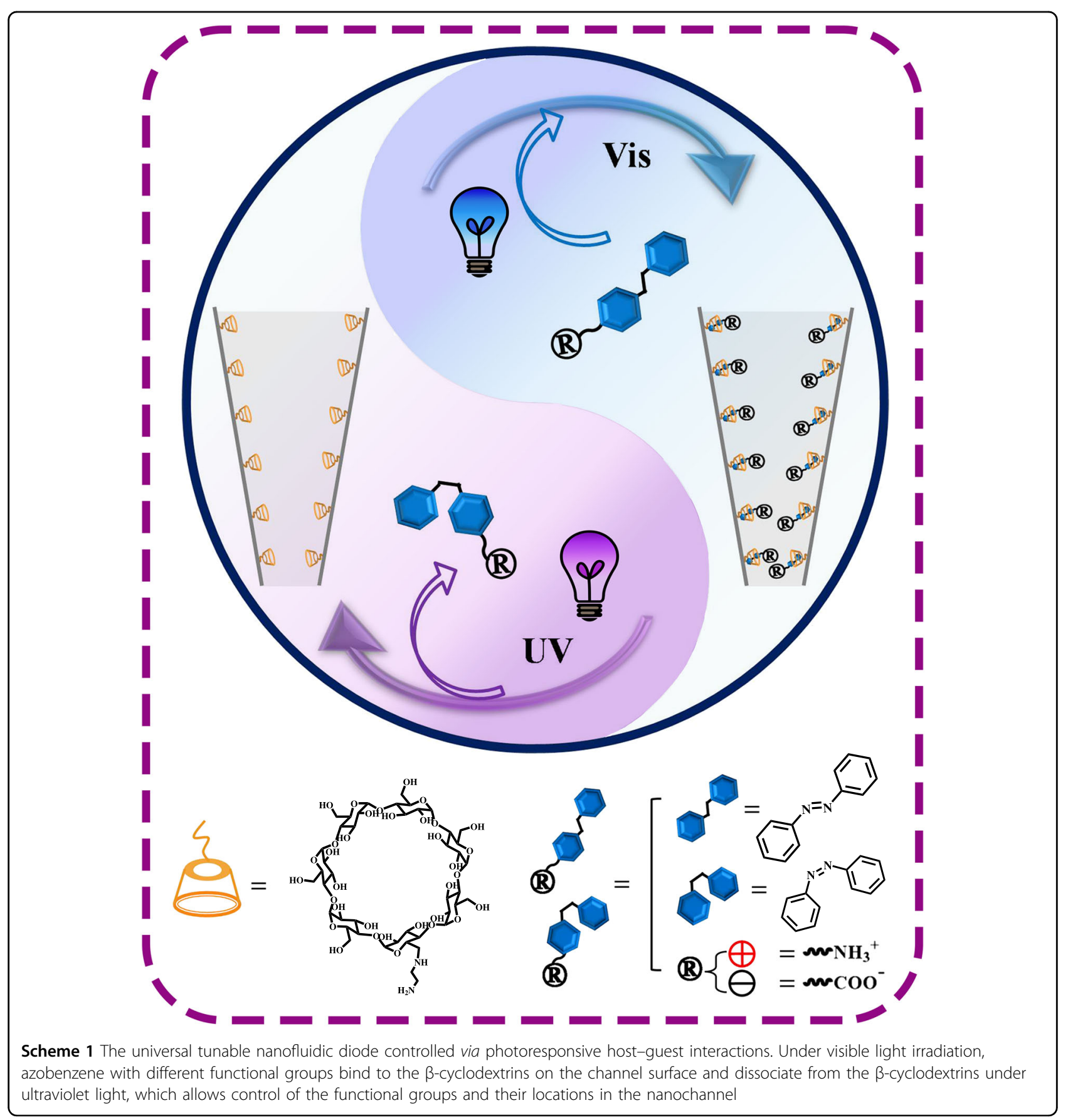




\section{Materials and methods Materials}

PI (GSI, Darmatadt, Germany, $12 \mu \mathrm{m}$ thick) film, potassium chloride $(\mathrm{KCl}), \quad N$-hydroxysulfosuccinimide (NHSS), 1-ethyl-3-(3-dimethyllaminopropyl) carbodiimide, (EDC), potassium iodide (KI), sodium hypochlorite (NaClO, 13\%), 4-(phenylazo)benzoic acid (Azo-COOH), and Azo were purchased from Sinopharm Chemical Reagent Beijing Co., Ltd. (SCRC, China). Mono-6-amino- $\beta$ cyclodextrin (EDA- $\beta-C D)$ and ((phenyldiazenyl)phenoxy) propan-1-amine (Azo- $\left.\mathrm{NH}_{2}\right)$ were synthesized according to the reported protocol (Supporting Note 1 and Figure S1). ${ }^{42}$ All solutions were prepared in MilliQ water $(18.2 \mathrm{M} \Omega$ ).

\section{Fabrication of nanochannel-based nanofluidic diodes}

The single conical PI nanochannel was prepared via an asymmetric track-etched technique with a single-ion track (Supporting Note 2 for details). In brief, the ion track PI membrane was mounted between two chambers of a homemade conductivity cell. The membrane was etched using etching solution $(\mathrm{NaClO}, 13 \%)$ in one chamber and stopping solution $(1 \mathrm{M} \mathrm{KI})$ in the other chamber at $60^{\circ} \mathrm{C}$. After the etching process, EDA- $\beta$-CDs were successively immobilized on the nanochannel surface by a conventional EDC/NHSS coupling reaction with the carboxyl groups on the nanochannel surface. Prior to the reaction, the carboxyl groups on the PI film were activated in $1 \mathrm{~mL}$ of an aqueous solution containing $15 \mathrm{mg}$ EDC and $3 \mathrm{mg}$ NHSS for $1 \mathrm{~h}$ at room temperature. Then, the PI film was washed and immersed in $10 \mathrm{mM}$ EDA- $\beta-C D$ aqueous solution overnight. Finally, the modified film was washed several times with distilled water.

\section{Characterization of the nanofluidic diode system}

The asymmetric ion transport properties of the nanofluidic system were evaluated by the currentvoltage $(I-V)$ curves with a Keithley 6487 picoammeter (Keithley Instruments, Cleveland, $\mathrm{OH}$ ) in $0.1 \mathrm{M} \mathrm{KCl}$. A single channel in the center of the PI membrane was mounted between the two chambers of the etching cell. $\mathrm{Ag} / \mathrm{AgCl}$ electrodes were used to apply a transmembrane potential. The main transmembrane potential used in this work was a scanning voltage that varied from $-2 \mathrm{~V}$ to $+2 \mathrm{~V}$. The anode faced the large opening (base) of the nanochannel, and the cathode faced the small opening (tip) of the nanochannel. All measurements were carried out at room temperature. The nanofluidic diodes were characterized by SEM, XPS, and UV-vis spectroscopy measurements (Supporting Note 2, Supporting Note 5, and Supporting Note 6 for details).

\section{Asymmetric modification}

The asymmetric modification setup is shown in Figure S9. By changing the types and the position of the Azo solution, two asymmetric states, “ $\mathrm{T}(+)-\mathrm{B}(-)$ ” and “ $\mathrm{T}$ $(-)-\mathrm{B}(+)$ ", were formed. The intensity of the light in the center of the setup was $22 \mathrm{~mW}$.

\section{Theoretical simulation of ion transport}

The ion rectification phenomenon was theoretically investigated with the commercial finite-element software package COMSOL (version 5.0) Multiphysics using the "electrostatics (Poisson equation)" and "Nernst-Planck without Electroneutrality" modules (Supporting Note 7 for details). Four states (positively charged nanochannel, negatively charged nanochannel, $\mathrm{T}(+)-\mathrm{B}(-)$ and $\mathrm{T}(-)-\mathrm{B}$ $(+)$ were theoretically investigated using the above method.

\section{Results and discussion}

The single conical nanochannel in this work was fabricated through UV treatment and chemical etching on an ion-tracked PI membrane. ${ }^{43,44}$ The diameter of the large opening (base side) of the conical nanochannel was $\sim 400 \mathrm{~nm}$, whereas the diameter of the narrow opening (tip side) was calculated to be $\sim 20 \mathrm{~nm}$ (Supporting Note 2 and Figure S2). Resulting from the asymmetric structure of the nanochannel and the deprotonation of the $-\mathrm{COOH}$ groups on the etched surface, the rectification of the conical nanochannel was obtained by characterizing the asymmetric $I-V$ curve with a positive rectification degree $\left(\mathrm{pR}, \mathrm{pR}=\log \left[\left|\mathrm{I}_{-2 \mathrm{~V}} / \mathrm{I}_{+2 \mathrm{~V}}\right|\right]\right.$, where the base number is 10 and $\mathrm{I}_{-2 \mathrm{~V}}$ and $\mathrm{I}_{+2} \mathrm{~V}$ represent the current at $-2 \mathrm{~V}$ and + $2 \mathrm{~V}$, respectively). Here, the rectifying direction with positive $\mathrm{pR}$ was defined as the forward rectifying direction, and vice versa.

EDA- $\beta$-CDs, as intermediates, were grafted into the nanochannel using a well-studied EDC-NHSS coupling reaction. The ion transport property of the nanochannel before and after modification with $\beta$-CDs was characterized by transmembrane ion current measurements in 0.1 $\mathrm{M} \mathrm{KCl}$ solutions of different $\mathrm{pH}$ values. After modification with $\beta$-CDs, owing to the reduction of effective negative charges on the surface, the ion current across the channel was reduced from $-25.3 \mathrm{nA}$ to $-5.3 \mathrm{nA}(\mathrm{pH} 7)$, and the corresponding $\mathrm{pR}$ was reduced from 1.18 to 0.89 ( $\mathrm{pH} 7$ ) (Figure S3). The results of the current measurements along with the X-ray photoelectron spectroscopy (XPS) analysis revealed that $\beta$-CDs were modified onto the surface of the nanochannel (Figure S4 and Figure S5).

Based on the $\beta$-CD-immobilized nanochannel, (Azo- $\mathrm{NH}_{2}, 10 \mathrm{mM}$ ) was induced through host-guest interactions under visible light irradiation. A bidirectional rectification behavior was observed by measuring the transmembrane ion current in $0.1 \mathrm{M} \mathrm{KCl}$ solution of different $\mathrm{pH}$ values. In acidic electrolytes ( $\mathrm{pH} 2-3$ ), a backward rectification was observed, where the ion currents at positive voltages were higher than those at 


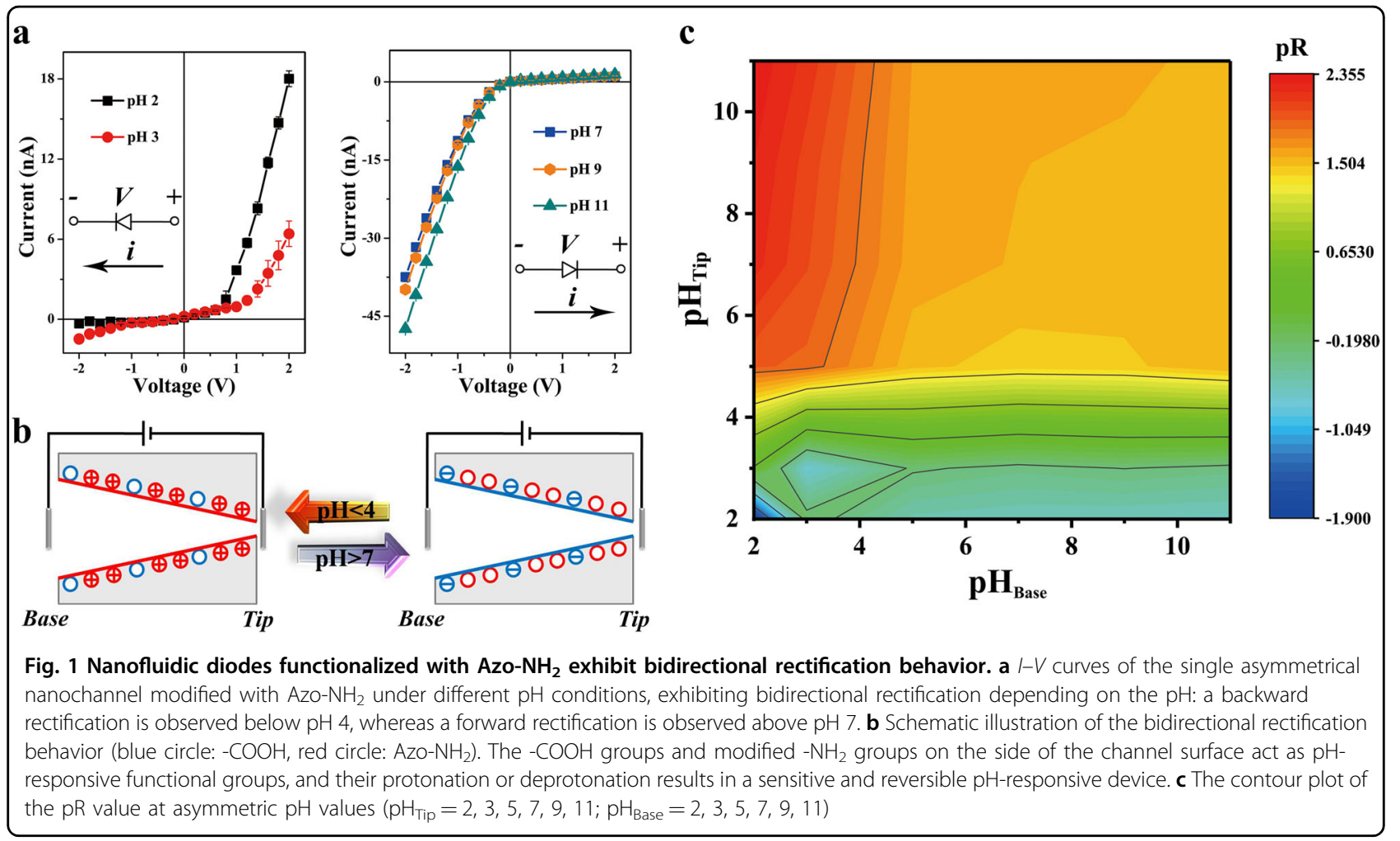

negative voltages (Fig. 1a, left), whereas the system changed into a forward rectification state and showed the opposite phenomenon in alkaline solution $(\mathrm{pH} 7-11)$ (Fig. 1a, right). This could be ascribed to changes in the effective pore size, wettability, and surface charge induced by the plugged Azo- $\mathrm{NH}_{2}{ }^{17,45,46}$ Owing to the protonation of Azo- $\mathrm{NH}_{2}$ and unreacted - $\mathrm{COOH}$, pR was $<0$ in solutions with $\mathrm{pH}<4$. However, when the environmental $\mathrm{pH}$ was above 7 , pR was $>0$ because of the deprotonation of groups on the surface (Fig. 1b).

To further investigate how the $\mathrm{pH}$ affects the charge status of the nanochannel as well as ion transport, $I-V$ curves were measured in $0.1 \mathrm{M} \mathrm{KCl}$ solution at a series of asymmetric $\mathrm{pH}$ values, and the corresponding $\mathrm{pR}$ values were calculated, which are summarized in Fig. 1c. The results show that the $\mathrm{pH}$ of the electrolyte solution has a significant effect on the rectification of the nanochannel. The $\mathrm{pR}$ value can be divided into the following three conditions: (a) approaching 0 (weak area: green in Fig. 1c), showing almost no rectification; (b) $>0$ (strong area with forward rectification: red and orange in Fig. 1c), which means $\left|\mathrm{I}_{-2} \mathrm{v} / \mathrm{I}_{+2} \mathrm{v}\right|>1$; and (c) $<0$ (strong area with backward rectification: blue in Fig. 1c), which means $\mid \mathrm{I}_{-2}$ $\mathrm{v} / \mathrm{I}_{+2} \mathrm{v} \mid<1$. When $\mathrm{pH}_{\text {Tip }}(\mathrm{pH}$ near the tip side of the nanosystem) is in the range of $7-11$ and $\mathrm{pH}_{\text {Base }}(\mathrm{pH}$ near the base side of the nanosystem) is below 4 , pR is located inside the strong area with values above 2 , and the corresponding rectification ratios are higher than 100 . Under such conditions, the functional groups on the tip side (pH 7-11) are deprotonated, and the functional groups on the base side $(\mathrm{pH}<4)$ are protonated. The asymmetric geometry along with the asymmetric charge distribution of the channel worked simultaneously and thus achieved very high rectification ratios. On the other hand, the $\mathrm{pR}$ value did not vary much over a long and narrow area $\left(\mathrm{pH}_{\text {Tip }}<3\right.$ and $\left.\mathrm{pH}_{\text {Base }} 5 \sim 11\right)$ with values below -0.6 , which showed that the states on the basic side had a relatively small impact on the whole system.

In addition, the nanofluidic diode shows good reversibility owing to the photocontrolled host-guest interaction between $\beta-C D$ and Azo- $\mathrm{NH}_{2}$. After treating $\beta-C D-$ immobilized nanochannels with Azo- $\mathrm{NH}_{2}$ under irradiation with visible light, Azo groups change into a trans conformation and bind with $\beta$-CDs, causing an increase in the positive charges; thus, the ion current at positive voltages $(+2 \mathrm{~V})$ increases from $0.065 \mathrm{nA}$ to $5.65 \mathrm{nA}$ at $\mathrm{pH}$ 3. Then, when irradiated by UV light, the Azo groups changed into a cis conformation and peeled off from the pore surface, which was confirmed by UV-vis spectrophotometry (Figure S6), and the corresponding ion current returned to $\sim 0.065 \mathrm{nA}$ (Fig. 2a). Moreover, the light-gated nanofluidic diode exhibits good stability and reversibility. Based on the $\beta-\mathrm{CD}$ molecules immobilized on the channel surface, reversible light-responsive switching between the two different ion conduction states of the nanofluidic diode was achieved by irradiating 


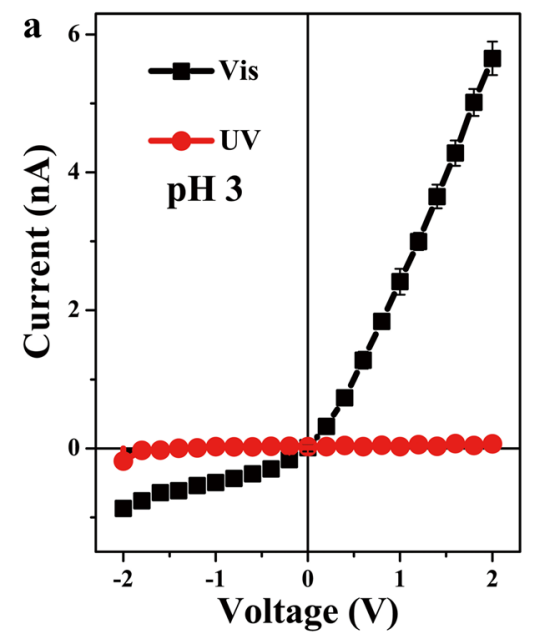

b

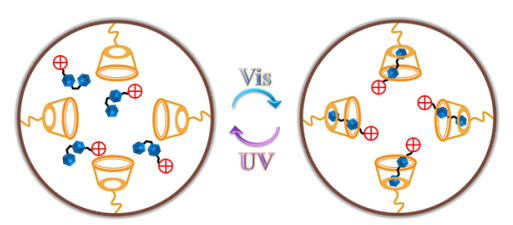

c

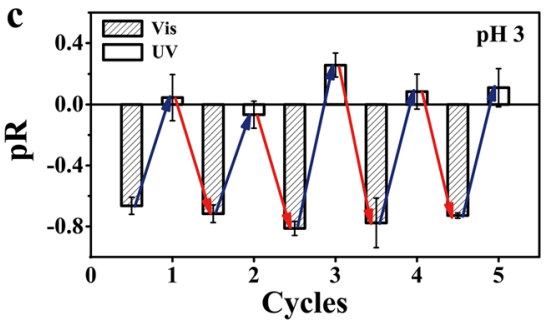

Fig. 2 Reversible functionalization of the nanofluidic diode controlled by supramolecular photochemistry. a $I-V$ curves of the nanochannels with Azo-NH $\mathrm{N}_{2}$ at pH 3 under irradiation with visible $(\boldsymbol{\square})$ and UV $(\mathbf{O})$ light. b Schematic illustration of the reversible light-responsive nanochannel. Using $\beta$-CDs as intermediates, Azo- $\mathrm{NH}_{2}$ could be bound to the surface of the channel under visible light and then released under UV light. c Reversible and recyclable switching of the $\mathrm{pR}$ value of the nanochannel at $\mathrm{pH} 3$ between two different rectification states by the inclusion (shaded column) and exclusion (unshaded column) of $\beta-\mathrm{CD}$ with Azo- $\mathrm{NH}_{2}$

visible and UV light (Fig. 2b), which can be expressed as the $\mathrm{pR}$ switching between $\sim-0.7$ and $\sim 0.1$ for at least five cycles at $\mathrm{pH} 3$ (Fig. 2c). Evidently, the nanofluidic diode possesses dual light and $\mathrm{pH}$ response and bidirectional adjustment, and these processes are reversible and recyclable owing to the light-responsive properties of Azo and the protonation and deprotonation of the functional groups on the surface of the channel under certain $\mathrm{pH}$ conditions.

Furthermore, our universal tunable nanofluidic diode system can be easily functionalized by other functional molecules. Here, Azo-COOH $(10 \mathrm{mM})$ was chosen to adjust the rectification of the system. Similar to Azo- $\mathrm{NH}_{2}$, Azo- $\mathrm{COOH}$ could also be attached to the pore surface through interactions with $\beta$-CDs, and the corresponding system exhibited dual light and $\mathrm{pH}$ response. After introducing Azo- $\mathrm{COOH}$, the ion current decreased from $-0.091 \mathrm{nA}$ to $-8.99 \mathrm{nA}$ at $-2 \mathrm{~V}$, and the $\mathrm{pR}$ value increased from -0.07 to 0.88 owing to the increase in $-\mathrm{COOH}$ groups on the surface (Fig. 3a). After exposure to UV light, the ion current at negative voltages was reduced because the Azo groups returned to the cis conformation and then dissociated from the $\beta$-CDs. In addition, the $\mathrm{pR}$ value of this system switched between $\sim 0.85$ and $\sim-0.15$ for at least five cycles, and no obvious change was observed in each state at pH 3 (Figure S7). This demonstrates good reversibility and cycling performance of the nanofluidic diode. Furthermore, the pH-responsive functional groups could precisely regulate the rectification property. With an increase in the $\mathrm{pH}$ of the electrolyte, the $\mathrm{pR}$ value of the system increased steady from -0.86 (at $\mathrm{pH}$ 2) to 1.45 (at $\mathrm{pH} 3$ ) to 1.79 (at $\mathrm{pH} 4$ ) and finally remained at $\sim 1.8$ (at $\mathrm{pH} 5,7,9)$ (Fig. 3b). The degree of protonation on the surface increased with increasing $\mathrm{pH}$, resulting in the gradual addition of negative charges on the channel surface and eventually to a saturated state. In addition to Azo-COOH and Azo- $\mathrm{NH}_{2}$, Azo was employed to functionalize the system as a control (Figure S8).

By controlling the distributions and density of available charge carriers inside the nanochannels, a system with flexible and tunable surface charges can be realized. The locations of the functional groups (Azo) in the nanochannel could be fine-tuned, and two asymmetric modified systems with light- and $\mathrm{pH}$-responsive properties were built (Fig. 4). To effectively construct a single nanochannel with controlled effective charges on its surface, Azo- $\mathrm{NH}_{2}$ and Azo-COOH, two types of Azo molecules with opposite charges in certain circumstances, were separately grafted onto the tip side (or base side) and the base side (or tip side) of the conical nanochannel bridged by $\beta$-CDs (Fig. 4a, S9).

After Azo- $\mathrm{NH}_{2}$ was bound to the tip side and Azo$\mathrm{COOH}$ to the base side through host-guest interactions with $\beta$-CDs, the system presented in the "T(+)-B(-)" state (left, Fig. 4a). In this state, the ion currents at positive voltages were much higher than those at negative voltages in $0.1 \mathrm{M} \mathrm{KCl}$ solution at $\mathrm{pH} 2$ owing to protonation of the $-\mathrm{NH}_{2}$ groups on the tip side (Figure S10a). To investigate the influence of $\mathrm{pH}$ on the above system, $I-V$ curves in $0.1 \mathrm{M} \mathrm{KCl}$ solution at asymmetric $\mathrm{pH}$ values of the two states were explored. It is worth noting that there are three sections in the contour plot: (a) approaching 0 (weak area: green in Fig. 4b), showing almost no rectification; (b) $>0$ (strong area with forward rectification: red 

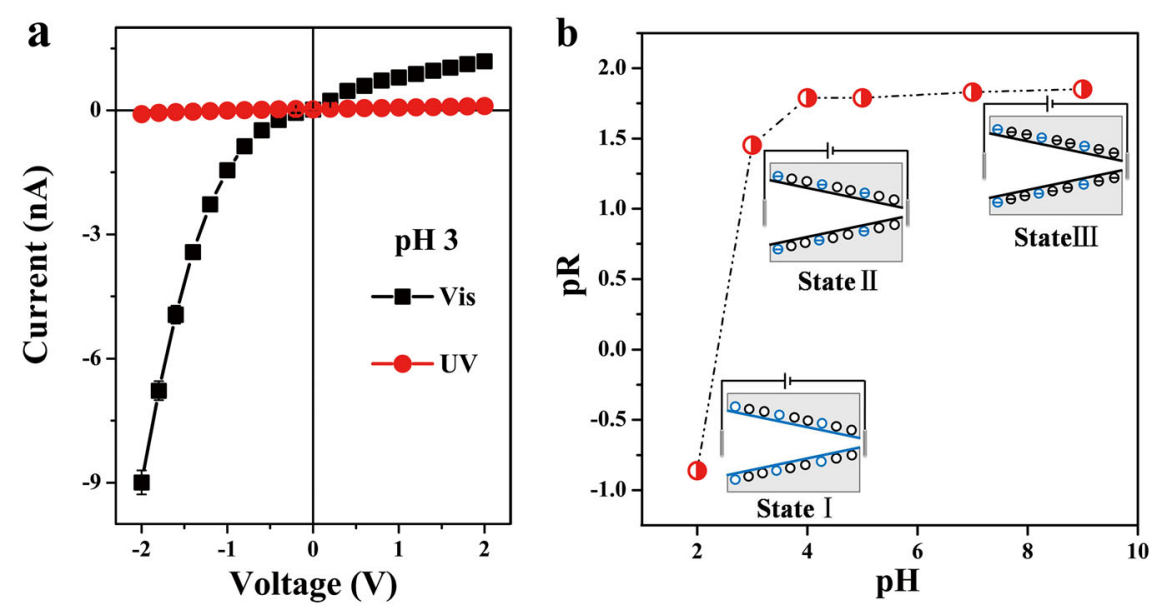

Fig. 3 Dual light and pH response of the Azo-COOH-modified nanochannel. a $1-V$ curves of the nanochannel with Azo-COOH after irradiation with visible light $(\mathbf{\square})$ and UV light $(\mathbf{O})$ at $\mathrm{pH}$ 3. b The pR values of the Azo- $\mathrm{COOH}$ modified channel at different pH values (from 2 to 9) undergo three main states; the value first increases in negative charge and then reaches a saturated state (blue circle: - $\mathrm{COOH}$, black circle: $\mathrm{AzO}-\mathrm{COOH}$ )

a

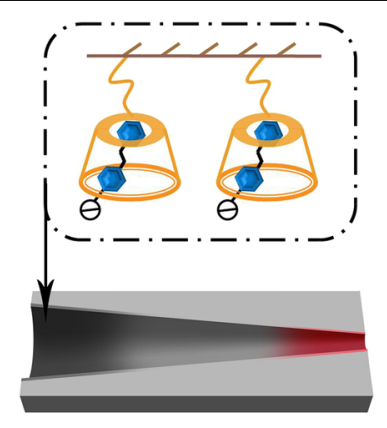

b

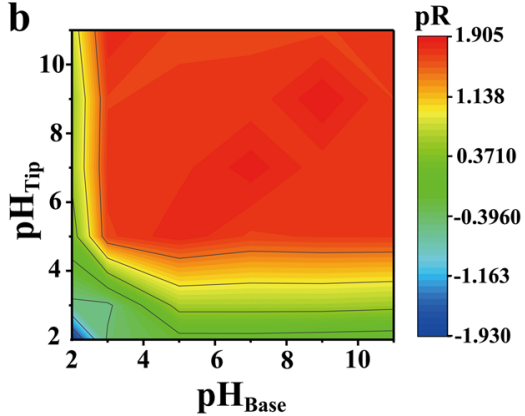

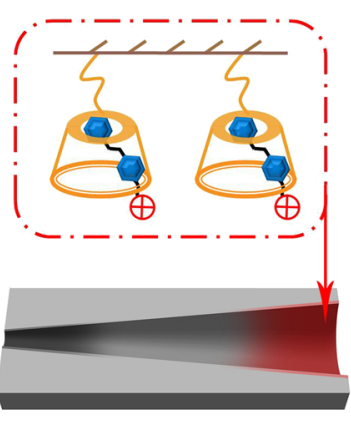

c

c

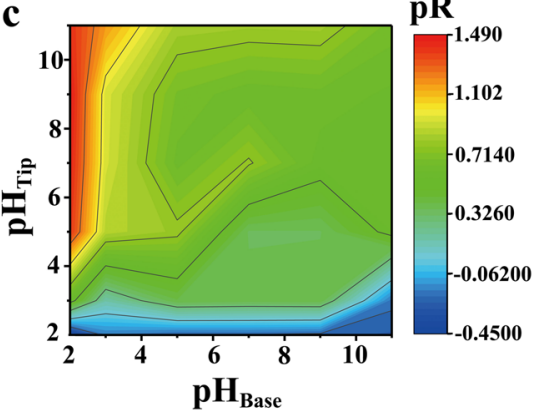

Fig. 4 Nanofluidic diodes with an asymmetric structure and chemistry. a Schematic illustration of the two states of the asymmetric modification: in the "T(+)-B(-)" state, Azo- $\mathrm{NH}_{2}$ was introduced on the tip side and $\mathrm{Azo}-\mathrm{COOH}$ on the base side, and in the "T(-)- $\mathrm{B}(+)$ " state, Azo-COOH was introduced on the tip side and $\mathrm{Azo}-\mathrm{NH}_{2}$ on the base side. $\mathbf{b}$ Contour plot of the $\mathrm{pR}$ values of the "T(+)- $\mathrm{B}(-)^{\prime}$ " state at asymmetric $\mathrm{pH}$ values $(\mathrm{pH}$ Tip $=2$, $\left.3,5,7,9,11 ; \mathrm{pH}_{\text {Base }}=2,3,5,7,9,11\right)$. c Contour plot of the $\mathrm{pR}$ values of the "T(-)-B(+)" state under asymmetric $\mathrm{pH}$ values $\left(\mathrm{pH}_{\text {Tip }}=2,3,5,7,9,11\right.$; $\left.\mathrm{pH}_{\text {Base }}=2,3,5,7,9,11\right)$

in Fig. 4b), which means $\left|I_{-2} v\right|>\left|I_{+2} v\right|$; and (c) $<0$ (strong area with backward rectification: blue in Fig. 4b), which means $\left|\mathrm{I}_{-2} \mathrm{v}\right|<\left|\mathrm{I}_{+2} \mathrm{v}\right|$. When $\mathrm{pH}_{\text {Tip }} 5 \sim 11$ and $\mathrm{pH}_{\text {Base }} 3 \sim 11$, the system was located in a large strong area with $\mathrm{pR}>1.6$, which means the relevant rectification ratios were $>44$. By introducing negative charges on the base side, the system with strong forward rectification extended over a long range compared with the system functionalized with Azo- $\mathrm{NH}_{2}$ (Fig. 1c). When $\mathrm{pH}_{\text {Tip }}<3$ and $\mathrm{pH}_{\text {Base }}<3$, the system showed strong backward rectification with $\mathrm{pR}<-0.43$ and a minimum $\mathrm{pR}$ of -1.93 .

In the " $\mathrm{T}(-)-\mathrm{B}(+)$ " state (right, Fig. 4a), the ion current at negative voltage decreased much after modification in $0.1 \mathrm{M} \mathrm{KCl}$ solution at $\mathrm{pH} 11$ (Figure S10b), and there are 
two long and narrow zones with opposite rectification directions in the contour plot in Fig. 4c. The forward ( $\mathrm{pR}$ $>0.85)$ and backward $(\mathrm{pR}<-0.17)$ rectification zones were located in the range of $\left(\mathrm{pH}_{\text {Tip }} 5 \sim 11, \mathrm{pH}_{\text {Base }}<3\right)$ and $\left(\mathrm{pH}_{\text {Tip }}<2, \mathrm{pH}_{\text {Base }} 2 \sim 11\right)$, respectively. When $\mathrm{pH}_{\text {Tip }}$ was decreased from 11 to 2 and $\mathrm{pH}_{\text {Base }}$ was increased from 2 to 11 , the system gradually stepped from the forward rectification area to the backward rectification area, which means the $\mathrm{pR}$ transforms from positive to negative (Fig. 4c). As shown in Fig. 4c, the distribution of $\mathrm{pR}$ is unlike the situation in the " $\mathrm{T}(+)-\mathrm{B}(-)$ " state (Fig. $4 \mathrm{~b}$ ), indicating the capability of tuning the ion transport by replacing the surface functional groups in the proposed system. In addition, both nanofluidic systems in the " $\mathrm{T}$ $(+)-\mathrm{B}(-)$ " and " $\mathrm{T}(-)-\mathrm{B}(+)$ " states show good reversibility and cycling performance (Figure S11).

To better understand the asymmetric geometry- and charge-dependent rectification behavior, finite-element simulations were performed to qualitatively describe the mechanism based on coupled PNP (Poisson-NernstPlanck) equations. ${ }^{47-50}$ The total length of all simulated models was uniformly set to $2000 \mathrm{~nm}$ (Supporting Note 7 and Figure S12). In Fig. 5a, the concentration profile shows that ions, mainly anions $\left(\mathrm{Cl}^{-}\right)$, accumulate in the positively charged nanochannel under a positive voltage bias. However, reversing the direction of the applied electric field drives the anions $\left(\mathrm{Cl}^{-}\right)$outward from the channel, causing the formation of a depletion zone with a low concentration profile. In contrast, the ions, mainly cations $\left(\mathrm{K}^{+}\right)$, accumulate strongly at negative voltage and deplete at positive voltage when the entire nanochannel is negatively charged (Fig. 5b). By introducing an asymmetric charge distribution, the concentration profiles change intensively. For the positively charged tip and negatively charged base (Fig. 5c) nanochannel, which is the " $\mathrm{T}(+)-\mathrm{B}(-)$ " state in Fig. 4a, the concentration profiles at $+2 \mathrm{~V}$ and $-2 \mathrm{~V}$ are strengthened compared with those in Fig. 5a. The reasons for the strengthened ion accumulation and depletion in this asymmetric geometry- and chargedependent nanochannel can be divided into three parts: (1) no external voltages were applied-the cations $\left(\mathrm{K}^{+}\right)$were mainly in the negatively charged base side, whereas the anions $\left(\mathrm{Cl}^{-}\right)$were enriched in the positively charged tip side; (2) positive voltages were applied (from the base side to the tip side) - both anions and cations were driven toward the junction, and after the system reached a steady state, the ions in the channel were enriched, causing a high ion current; and (3) negative voltages were applied (from the tip side to the base side) -both anions and cations migrate outward from the border area under opposite electrical fields, leading to a depletion of ions in the channel and causing a large decrease in the ion current. ${ }^{7,23}$ In contrast, a significant increase in the concentration profile was observed at -2 $\mathrm{V}$ (Fig. 5d) when the tip side was negatively charged and the base side was positively charged ("T $(-)-\mathrm{B}(+)$ " state). The simulated results agreed well with our experimental measurements and provided visual ion concentration distributions for analysis. The simulation shows that our designed replaceable functional group mechanism for rationally controlling the charge distribution can finely tune the ion transport property.

\section{Conclusion}

In summary, we successfully fabricated a $\mathrm{pH}$-regulated bidirectional nanofluidic diode by employing photoresponsive host-guest interactions based on a PI membrane. Through host-guest interactions between $\beta-C D$ and Azo, nanofluidic diodes with different functional groups

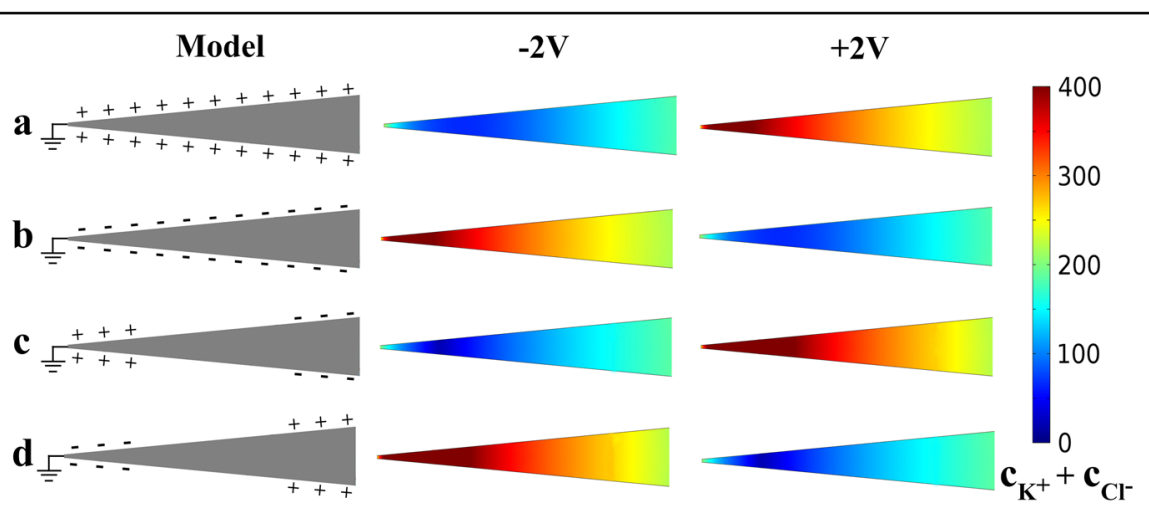

Fig. 5 Numerical simulation of the nanofluidic diode with varied surface charge and the corresponding concentration profile of the ion distribution in the nanochannel at $+\mathbf{2} \mathbf{V}$ and $-\mathbf{2} \mathbf{V}$. a Positively charged nanochannels result in ion (mainly anions) accumulation at $+2 \mathrm{~V}$ and depletion at $-2 \mathrm{~V}$. $\mathbf{b}$ Negatively charged nanochannels result in ion (mainly cations) accumulation at $+2 \mathrm{~V}$ and depletion at $-2 \mathrm{~V}$. $\mathbf{c}$ lons deplete strongly at $-2 \mathrm{~V}$ and accumulate strongly at $+2 \mathrm{~V}$ when the tip side is positively charged and the base side is negatively charged. $\mathbf{d}$ lons accumulate strongly at $-2 \mathrm{~V}$ and deplete strongly at $+2 \mathrm{~V}$ when the tip side is negatively charged and the base side is positively charged 
(Azo-COOH and Azo- $\mathrm{NH}_{2}$ ) could be prepared as needed, and the degree of rectification could be adjusted with high precision by tuning the $\mathrm{pH}$. Furthermore, this work presents great potential in smart device establishment by introducing host-guest chemistry into nanofluidics. The dual light and $\mathrm{pH}$ response of the PI single nanofluidic systems may allow potential applications in fields such as photosensitive nanofluidic devices, light-controlled drug transport, ${ }^{51-53} \mathrm{pH}$-activated drug release and devices for optical information storage. In addition, the reversible and bidirectional switching characteristics allow further development in nanofluidic logic devices. ${ }^{54-57}$

\section{Acknowledgements}

We thank the Material Science Group of GSI (Darmstadt, Germany) for providing the ion-irradiated samples. This work was supported by the National Key R\&D Program of China (2017YFA0206904, 2017YFA0206900), the National Natural Science Foundation of China (21625303, 51673206, 21434003), and the Key Research Program of the Chinese Academy of Sciences (QYZDY-SSWSLH014)

\section{Author details}

${ }^{1}$ CAS Key Laboratory of Bioinspired Materials and Interfacial Science, Technical Institute of Physics and Chemistry, Chinese Academy of Sciences, Beijing 100190, China. ${ }^{2}$ School of Future Technology, University of Chinese Academy of Science, Beijing 100049, China. ${ }^{3}$ Key Laboratory of Bio-Inspired Smart Interfacial Science and Technology of Ministry of Education School of Chemistry and Environment, Beihang University, Beijing 100190, China

\section{Conflict of interest}

The authors declare that they have no conflict of interest.

\section{Publisher's note}

Springer Nature remains neutral with regard to jurisdictional claims in published maps and institutional affiliations.

Supplementary information is available for this paper at https://doi.org/ 10.1038/s41427-018-0079-5.

Received: 1 April 2018 Revised: 2 July 2018 Accepted: 3 July 2018. Published online: 31 August 2018

\section{References}

1. Doyle, D. A. et al. The structure of the potassium channel: molecular basis of $\mathrm{K}^{+}$conduction and selectivity. Science 280, 69-77 (1998).

2. Gouaux, E. \& Mackinnon, R. Principles of selective ion transport in channels and pumps. Science 310, 1461-1465 (2005).

3. Xu, J. \& Lavan, D. A. Designing artificial cells to harness the biological ion concentration gradient. Nat. Nanotechnol. 3, 666-670 (2008).

4. Fyles, T. M. Synthetic ion channels in bilayer membranes. Chem. Soc. Rev. 36, 335-347 (2007).

5. Kumar, B. V., Rao, K. V., Sampath, S., George, S. J. \& Eswaramoorthy, M. Supramolecular gating of ion transport in nanochannels. Angew. Chem. Int. Ed. 53, 13073-13077 (2014).

6. Yamaguchi, $\mathrm{H}$. et al. Photoswitchable gel assembly based on molecular recognition. Nat. Commun. 3, 603-607 (2012).

7. Guan, W., Fan, R. \& Reed, M. A. field-effect reconfigurable nanofluidic ionic diodes. Nat. Commun. 2, 506-513 (2011).

8. Rao, S. et al. A light-powered bio-capacitor with nanochannel modulation Adv. Mater. 26, 5846-5850 (2014).

9. Sun, Y. et al. A light-regulated host-guest-based nanochannel system inspired by channelrhodopsins protein. Nat. Commun. 8, 260-266 (2017).
10. Li, R., Fan, X., Liu, Z. \& Zhai, J. Smart bioinspired nanochannels and their applications in energy-conversion systems. Adv. Mater. 29, 1702983-1702989 (2017).

11. Chen, Z. et al. A novel biomimetic logic gate for sensitive and selective detection of $\mathrm{Pb}(\mathrm{II})$ base on porous alumina nanochannels. Electrochem. Commun. 60, 83-87 (2015).

12. Zhai, Q. et al. Biomimetic nanopore for sensitive and selective detection of $\mathrm{Hg}$ (II) in conjunction with single-walled carbon nanotubes. J. Mater. Chem. B 2, 6371-6377 (2014)

13. Cheng, L. \& Guo, L. J. Nanofluidic diodes. Chem. Soc. Rev. 39, $923-938$ (2010).

14. He, Y. et al. Tuning transport properties of nanofluidic devices with local charge inversion. J. Am. Chem. Soc. 131, 5194-5202 (2009).

15. Perez-Mitta, G., Marmisolle, W. A., Trautmann, C., Toimil-Molares, M. E. \& Azzaroni, O. An all-plastic field-effect nanofluidic diode gated by a conducting polymer layer. Adv. Mater. 29, 1700972-1700978 (2017).

16. Siwy, Z. \& Fulinski, A. A nanodevice for rectification and pumping ions. Am. J. Phys. 72, 567-574 (2004).

17. Vlassiouk, I. \& Siwy, Z. S. Nanofluidic diode. Nano. Lett. 7, 552-556 (2007).

18. Siwy, Z. S. Ion-current rectification in nanopores and nanotubes with broken symmetry. Adv. Funct. Mater. 16, 735-746 (2006).

19. Ali, M. et al. Single cigar-shaped nanopores functionalized with amphoteric amino acid chains: experimental and theoretical characterization. ACS Nano 6 , 3631-3640 (2012).

20. Wang, R. et al. Temperature-sensitive artificial channels through pillar[5]arenebased host-guest interactions. Angew. Chem. Int. Ed. 129, 5378-5382 (2017).

21. Liu, Q. et al. A fluoride-driven ionic gate based on a 4-aminophenylboronic acid-functionalized asymmetric single nanochannel. ACS Nano 8, 12292-12299 (2014).

22. Perez-Mitta, G., Marmisolle, W. A., Trautmann, C., Eugenia Toimil-Molares, M., \& Azzaroni, O. Nanofluidic diodes with dynamic rectification properties stemming from reversible electrochemical conversions in conducting polymers. J. Am. Chem. Soc. 137, 15382-15385 (2015).

23. Zhang, Z. et al. Asymmetric multifunctional heterogeneous membranes for $\mathrm{pH}$ - and temperature-cooperative smart ion transport modulation. Adv. Mater. 28, 9613-9619 (2016)

24. Zhang, $\mathrm{M}$. et al. Light and $\mathrm{pH}$ cooperative nanofluidic diode using a spiropyran-functionalized single nanochannel. Adv. Mater. 24, 2424-2428 (2012).

25. Ali, M. et al. Optical gating of photosensitive synthetic ion channels. Adv. Funct. Mater. 22, 390-396 (2012).

26. Wang, Z. et al. $\mathrm{pH}$ - and light-regulated ion transport in hourglass shaped $\mathrm{Al}_{2} \mathrm{O}_{3}$ nanochannels patterned with N719 and APTES. RSC Adv. 6, 63652-63659 (2016).

27. Xie, G. et al. A bioinspired switchable and tunable carbonate-activated nanofluidic diode based on a single nanochannel. Angew. Chem. Int. Ed. 54, 13664-13668 (2015)

28. Xu, Y., Sui, X., Guan, S., Zhai, J. \& Gao, L. Olfactory sensory neuron-mimetic $\mathrm{CO}_{2}$ activated nanofluidic diode with fast response rate. Adv. Mater. 27, 1851-1855 (2015).

29. Xu, Y., Sui, X., Jiang, J., Zhai, J. \& Gao, L. Smooth muscle cell-mimetic COregulated ion nanochannels. Adv. Mater. 28, 10780-10785 (2016).

30. Huang, Z. et al. Supramolecular polymerization promoted and controlled through self-sorting. Angew. Chem. Int. Ed. 53, 5351-5355 (2014).

31. Kang, Y. et al. Controllable supramolecular polymerization promoted by host enhanced photodimerization. ACS Macro Lett. 5, 1397-1401 (2016).

32. Chen, L., Bai, H., Xu, J.F., Wang, S. \& Zhang, X. Supramolecular porphyrin photosensitizers: controllable disguise and photoinduced activation of. antibacterial behavior. ACS Appl. Mater. Interfaces 9, 13950-13957 (2017).

33. Hatano, J., Okuro, K. \& Aida, T. Photoinduced bioorthogonal 1,3-dipolar polycycloaddition promoted by oxyanionic substrates for spatiotemporal operation of molecular glues. Angew. Chem. Int. Ed. 55, 193-198 (2016).

34. Izquierdo-Serra, M. et al. Optical control of endogenous receptors and cellular excitability using targeted covalent photoswitches. Nat. Commun. 7 $12221-12233$ (2016).

35. Liu, X., Xu, J.F., Wang, Z. \& Zhang, X. Photo-responsive supramolecular polymers synthesized by olefin metathesis polymerization from supramonomers. Polym. Chem. 7, 2333-2336 (2016).

36. Thevarpadam, J. et al. Photoresponsive formation of an intermolecular minimal G-quadruplex motif. Angew. Chem. Int. Ed. 55, 2738-2742 (2016).

37. Peng, L. et al. Reversible phase transfer of nanoparticles based on photoswitchable host-guest chemistry. ACS Nano 8, 2555-2561 (2014). 
38. Wang, Y., Ma, N., Wang, Z. \& Zhang, X. Photocontrolled reversible supramolecular assemblies of an azobenzene-containing surfactant with alphacyclodextrin. Angew. Chem. Int. Ed. 46, 2823-2826 (2007).

39. Xiao, X., Nie, G., Zhang, X., Tian, D. \& Li, H. Protein adsorption switch constructed by a pillar[5]arene-based host-guest interaction. Chem. Eur. J. 22, 941-945 (2016)

40. Yang, L., Bai, Y., Tan, X., Wang, Z. \& Zhang, X. Controllable supramolecular polymerization through host-guest interaction and photochemistry. ACS Macro Lett. 4, 611-615 (2015)

41. Xie, G. et al. Light- and electric-field-controlled wetting behavior in nanochannels for regulating nanoconfined mass transport. J. Am. Chem. Soc. 140 4552-4559 (2018).

42. Liu, Y. Y., Fan, X. D. \& Gao, L. Synthesis and characterization of beta-cyclodextrin based functional monomers and its copolymers with $\mathrm{N}$-isopropylacrylamide. Macromol. Biosci. 3, 715-719 (2003).

43. Apel, P. Y., Korchev, Y. E. \& Siwy, Z. Diode-like single-ion track membrane prepared by electro-stopping. Nucl. Instrum. Methods Phys. Res. B 184, 337-346 (2001).

44. P., A. Track etching technique in membrane technology. Radiat. Meas. 34 559-566 (2001).

45. M., W. \& A., M. Chemically modified solid-state nanopores. Nano. Lett. 7, 1580-1585 (2007).

46. Powell, M. R., Cleary, L., Davenport, M., Shea, K. J. \& Simy, Z. S. Electric-fieldinduced wetting and dewetting in single hydrophobic nanopores. Nat. Nanotechnol. 6, 798-802 (2011).

47. Cervera, J., Schiedt, B., Neumann, R., Mafe, S. \& Ramirez, P. Ionic conduction, rectification, and selectivity in single conical nanopores. J. Chem. Phys. 124, 104706-104721 (2006)
48. Lan, W.-J., Holden, D. A. \& White, H. S. Pressure-dependent ion current rectification in conical-shaped glass nanopores. J. Am. Chem. Soc. 133, 13300-13303 (2011).

49. White, H. S. \& Bund, A. Mechanism of electrostatic gating at conical glass nanopore electrodes. Langmuir 24, 12062-12067 (2008).

50. White, H. S. \& Bund, A. Ion current rectification at nanopores in glass membranes. Langmuir 24, 2212-2218 (2008).

51. Giri, S., Trewyn, B. G., Stellmaker, M. P. \& Lin, V. S. Y. Stimuli-responsive controlled-release delivery system based on mesoporous silica nanorods capped with magnetic nanoparticles. Angew. Chem. Int. Ed. 44, 5038-5044 (2005).

52. Sparreboom, W., van den Berg, A.\& Eijkel, J. C. T. Principles and applications of nanofluidic transport. Nat. Nanotechnol. 4, 713-720 (2009).

53. van der Heyden, F. H. J., Bonthuis, D. J., Stein, D., Meyer, C. \& Dekker, C. Power generation by pressure-driven transport of ions in nanofluidic channels. Nano. Lett. 7, 1022-1025 (2007).

54. Ali, M., Mafe, S., Ramirez, P., Neumann, R. \& Ensinger, W. Logic gates using nanofluidic diodes based on conical nanopores functionalized with polyprotic acid chains. Langmuir 25, 11993-11997 (2009).

55. Guliyev, R., Ozturk, S., Kostereli, Z. \& Akkaya, E. U. From virtual to physical: integration of chemical logic gates. Angew. Chem. Int. Ed. 50, 9826-9831 (2011).

56. Gupta, T. \& van der Boom, M. E. Redox-active monolayers as a versatile platform for integrating boolean logic gates. Angew. Chem. Int. Ed. 47, 5322-5326 (2008)

57. Mafe, S., Manzanares, J. A. \& Ramirez, P. Gating of nanopores: modeling and implementation of logic gates. J. Phys. Chem. C 114, 21287-21290 (2010). 